\title{
Three-phase electrical signals analysis for mechanical faults monitoring in rotating machine systems
}

\author{
Georgia Cablea $^{\mathrm{a}, \mathrm{b}, *}$, Pierre Granjon ${ }^{\mathrm{a}, \mathrm{b}}$, Christophe Bérenguer ${ }^{\mathrm{a}, \mathrm{b}}$ \\ ${ }^{a}$ Univ. Grenoble Alpes, GIPSA-Lab, F-38000 Grenoble, France \\ ${ }^{b}$ CNRS, GIPSA-Lab, F-38000 Grenoble, France
}

\begin{abstract}
The current paper proposes a method to detect mechanical faults in rotating machines using three-phase electrical currents analysis. The proposed fault indicator relies on the use of instantaneous symmetrical components (ISCs), followed by a demodulation step enhancing the small modulations generated in electrical signals by mechanical faults. The limitations due to the multi-component nature of electrical signals, as well as to the noise naturally present in the measured signals are studied and taken into account in order to elaborate a proper and efficient algorithm to compute a mechanical fault indicator. It is theoretically shown that the ISCs based approach results in an increase of the signal-tonoise ratio compared to a single-phase approach, finally leading to an improvement of early fault detection capabilities. This result is validated using both synthetic and experimental signals where the proposed method is used to detect bearing faults and the obtained results are compared to single-phase results.
\end{abstract}

Keywords: Instantaneous symmetrical components, three-phase electrical signals, electrical rotating machines, bearing faults, condition monitoring

\section{Introduction}

Nowadays, three-phase rotating machines are widely used in most industries like manufacturing plants (e.g. paper mills), transportation (e.g. electric vehicles) or power generation (e.g. wind turbines). Particularly, three-phase induction machines are most commonly used due to their robustness and lower price. Either used as electric generators to convert mechanical energy to electrical energy, or as electric motors to convert the energy in the other direction, three-phase rotating machines are a connecting component between a mechanical system and an electrical one. Such electro-mechanical systems are subject to both electrical and mechanical faults. In this paper the focus is set on mechanical faults generating load

\footnotetext{
*Corresponding author

Email addresses: Georgia.Cablea@gipsa-lab.grenoble-inp.fr (Georgia Cablea), Pierre.Granjon@gipsa-lab.grenoble-inp.fr (Pierre Granjon), Christophe.Berenguer@gipsa-lab.grenoble-inp.fr (Christophe Bérenguer)

Preprint submitted to Mechanical Systems and Signal Processing
}

January 19, 2017 
torque oscillations on the machine shaft. Such faults consist for example in bearing faults or gearbox faults, and can be located in the machine itself, as well as in a connected mechanical system, i.e. in the drive-train.

Several condition monitoring methods have been developed in order to detect mechanical faults using electrical quantities involved in such machines. In particular, condition monitoring through stator current analysis, also known as motor current signature analysis (MCSA), is now well developed for single-phase currents [1]. This method relies on the fact that such faults generate amplitude and phase modulations in stator currents $[2,3]$. One important point concerning single-phase approaches is that the information contained in each stator current is slightly different from phase to phase due to initial phase shifts, amplitude and/or phase unbalance, high frequency components, measurement noise, etc. Therefore, methods based on the analysis of single-phase currents use only one part of the whole information available in the three-phase system.

As a consequence, current research in the field of condition monitoring of three-phase systems focuses on developing methods taking into account three-phase quantities. In order to efficiently combine and process the information contained in the three phases as a whole, several three-phase transforms have been used such as the Fortescue transform [4] or the Clarke/Concordia transform [5]. In [6], all these transforms are shown to be equivalent, finally leading to the instantaneous symmetrical components (ISCs). More particularly, the positive-sequence ISC of the stator currents is generally demodulated to detect mechanical faults in three-phase rotating machines. For example, the so-called Extended Park's Vector Approach (EPVA) [7] computes the squared modulus of this component in order to estimate and analyze its instantaneous amplitude to detect bearing failures. In [8], the instantaneous frequency of the positive-sequence ISC is estimated and used to monitor mechanical faults inducing load-torque oscillations on the machine shaft. The positive-sequence ISC obtained with a Concordia transform is amplitude and frequency demodulated in [9], [10], [11] and [12], and the obtained results are compared to several other demodulation techniques. In all these studies, two major points have been identified as limitations. Firstly, while the noise naturally present in measured signals may hide incipient fault signatures and deteriorate early fault detection capabilities, none of the previous study investigate its specific influence on the ISCs. Secondly, the demodulation steps of the positive-sequence ISC proposed in the previous papers assume that this signal is a monocomponent analytic signal, which is generally false. Indeed, the presence of harmonics and/or electrical unbalance in three-phase signals leads to the presence of different components with positive and negative frequency in the positive-sequence ISC, and finally to extra-oscillations in the demodulated quantities. These problems have been identified and partly solved in $[13,14]$, where a technique dedicated to the adaptive estimation of the fundamental frequency of balanced or unbalanced three-phase signals has been proposed. Another interesting approach relying on a principal component analysis (PCA) directly applied to three-phase electric signals has been proposed in $[11,12]$. This method has been studied under the assumptions of three-phase signals unbalanced in amplitude only and without any additive noise, and properly demodulates the signals in this particular case. The main drawback of [11] is that the PCA method assumes monocomponent signals (no harmonics or other frequency components). 
The previous observations lead to the two major contributions of this paper. The first one is a detailed study of the content of the ISCs in terms of periodic components as well as noises. The second contribution is to propose a mechanical fault indicator relying on a correct implementation of an amplitude and phase demodulation of the positive-sequence ISC, even in the presence of harmonics, electrical unbalance, and white or colored additive noises in electrical data. Beyond these two contributions, another interesting point is also addressed: while for electrical faults the advantage of using symmetrical components methods for condition monitoring is stated as the separation of balanced and unbalanced components [15], what is the real advantage of using three-phase approaches over single-phase ones for mechanical faults detection? Some answers are provided to this important question throughout the current paper, where it is shown that the proposed method improves early faults detection capabilities by increasing the signal-to-noise ratio in the positive-sequence ISC compared to single-phase currents. This last result is confirmed by simulated and experimental results obtained by comparing the proposed mechanical fault indicator relying on a three-phase approach with the same indicator computed from single-phase currents only.

The next section of this paper gives the definition of the instantaneous symmetrical components, their interpretation and argues their advantages compared to single-phase quantities. In Section 3, a single-phase current model for mechanical fault signature in electrical signals is extended to three-phase current signals. A mechanical fault indicator relying jointly on the proposed three-phase signal model and on instantaneous symmetrical components is then developed with the corresponding estimation algorithm. Its performance is illustrated in section 4 on experimental data acquired at the stator of a three-phase generator located on a test-bench emulating a wind turbine, and compared to single-phase results. Finally, the last section of this paper presents the overall conclusions of this research work.

\section{Effect of the instantaneous symmetrical components transform}

In this section, as well as in the rest of the paper, a complex-valued three-phase signal model was used for the theoretical development, while a real-valued signal formulation was used for the simulations. The choice of using a $\mathbb{C}$-valued model is justified by the need for clarity and to simplify computations. However, since real world signals are $\mathbb{R}$-valued, such a formulation was used for the simulations.

\subsection{Three-phase electrical signal model}

In order to study the effect of the ISC transform on three-phase signals, we consider the three-phase currents signals formulation as in Equation (1), where the complex-valued formulation of the signals model was chosen in order to simplify further theoretical computations.

$$
\mathbf{i}(t)=\mathbf{a} \cdot e^{\mathbf{j} 2 \pi f_{0} t}+\mathbf{n}(t)
$$

In (1), the vector $\mathbf{i}(t)=\left[\begin{array}{lll}i_{1}(t) & i_{2}(t) & i_{3}(t)\end{array}\right]^{T}$ comprises the three current signals, the vector $\mathbf{a}=\left[\begin{array}{lll}A_{1} & A_{2} & A_{3}\end{array}\right]^{T}$ contains the complex-valued amplitudes (or phasors) of the 
signals around the fundamental frequency $f_{0}$ and $\mathbf{n}(t)=\left[\begin{array}{lll}n_{1}(t) & n_{2}(t) & n_{3}(t)\end{array}\right]^{T}$ contains the additive noise present in the three-phase currents. As formulated, the signal model contains a periodical part corresponding to the fundamental component and a random part describing the noise. For clarity, the formulation is done under the assumption of stationary signals and only the components around the fundamental frequency $f_{0}$ are considered. As it will be further shown, these assumptions do not constrain the validity of the results.

Ideal three-phase systems are perfectly balanced around their fundamental components, meaning all three signals have the same amplitude and a phase shift of $\frac{2 \pi}{3}$ between each other. In (1), this would lead to the vector a expressed as:

$$
\mathbf{a}=A\left[\begin{array}{lll}
1 & e^{-\mathrm{j} \frac{2 \pi}{3}} & e^{\mathrm{j} \frac{2 \pi}{3}}
\end{array}\right]^{T}, \text { with } A \in \mathbb{C}
$$

Real systems are never perfectly balanced and three-phase currents often contain an inherent unbalance described as small amplitude differences and phase shifts slightly different from the desired $\frac{2 \pi}{3}$ value. Thus, the complex amplitudes in a correspond to phasors of a threephase system with eventually unbalanced phases and amplitudes. The noise $n_{k}(t)$, with $k \in\{1,2,3\}$, appearing on each phase is considered to be uncorrelated with the noise on the other phases. This noise is also assumed second order stationary with a power spectral density (PSD) $S_{n_{k}}(f)$ not necessarily constant over $f$.

\subsection{Instantaneous symmetrical components}

Three-phase electrical systems are often analyzed with symmetrical components originally introduced by Fortescue in [4]. They allow to separate the balanced and unbalanced parts of three-phase signals at their fundamental frequency, and are used for example to detect winding faults in rotating machines [15]. Lyon generalized this approach in [16] by applying the same concepts to time-dependent variables. This leads to instantaneous symmetrical components (ISCs), further detailed in [6] and used in the rest of this paper. Applied to the three-phase currents defined in equation (1), the corresponding transform expresses as:

$$
\left[\begin{array}{c}
i_{+}(t) \\
i_{-}(t) \\
i_{0}(t)
\end{array}\right]=\underbrace{\frac{1}{3}\left[\begin{array}{ccc}
1 & a & a^{2} \\
1 & a^{2} & a \\
1 & 1 & 1
\end{array}\right]}_{\boldsymbol{F}^{-1}}\left[\begin{array}{l}
i_{1}(t) \\
i_{2}(t) \\
i_{3}(t)
\end{array}\right]
$$

where $a=e^{\mathrm{j} \frac{2 \pi}{3}}, \boldsymbol{F}^{-1}$ denotes the inverse of the Fortescue matrix [4], and the instantaneous symmetrical components of the original three-phase signal $\mathbf{i}(t)$ are:

- $i_{+}(t)$ the positive-sequence ISC,

- $i_{-}(t)$ the negative-sequence ISC,

- $i_{0}(t)$ the zero-sequence ISC. 
In the rest of this paper, the transform defined in Eq. (3) is named instantaneous symmetrical components transform or ISC transform.

If the transform in (3) is applied to perfectly balanced three-phase signals around the fundamental frequency (Equation (1) and taking (2) into account), the resulting positivesequence ISC can be expressed as:

$$
i_{+}(t)=A e^{\mathrm{j} 2 \pi f_{0} t}+n_{+}(t)
$$

In (4) the positive-sequence ISC contains a periodic component at the fundamental frequency and $n_{+}(t)$ is a linear combination of the noises present in the phase signals. Indeed, the component corresponding to the fundamental frequency of the positive-sequence ISC is an analytic signal under balanced conditions. For the negative- and zero-sequence ISCs the amplitude of the frequency component located at $f_{0}$ would be null.

\subsection{Separation of balanced and unbalanced parts}

The main objective of using this transform has been stated as the property of separating balanced and unbalanced components contained in a three-phase signal. Indeed, the balanced part of the original three-phase signal is isolated in the positive-sequence component, whereas the negative- and zero-sequence components contain its unbalanced parts. This specific property can be understood for example by interpreting (3) as a set of projections. By denoting the indices of the ISCs as $m \in\{+,-, 0\}$ and $\alpha_{m k}$ the coefficients of $\boldsymbol{F}^{-1}$ on line $m$ and column $k$, each ISC can be expressed as:

$$
i_{m}(t)=\sum_{k=1}^{3} \alpha_{m k} i_{k}(t)=\sum_{k=1}^{3}\left(\alpha_{m k}^{*}\right)^{*} i_{k}(t)=\left\langle\mathbf{i}(t), \boldsymbol{\alpha}_{m}^{*}\right\rangle
$$

where $^{*}$ denotes complex conjugation and $\left\langle\mathbf{i}(t), \boldsymbol{\alpha}_{m}^{*}\right\rangle$ is the inner product between vectors $\boldsymbol{\alpha}_{m}^{*}$ and $\mathbf{i}(t)$.

Eq. (5) shows that each ISC can be expressed as an inner product between the vector of original phase currents and the complex conjugate of the corresponding line of the inverse Fortescue matrix. Equivalently, this relation can be considered as a projection of the phase signals on new axes. As, for example, the complex conjugate of $\boldsymbol{\alpha}_{+}=\frac{1}{3}\left[\begin{array}{lll}1 & a & a^{2}\end{array}\right]$ describes a perfectly balanced system of three-phase signals in a positive sequence order, the obtained positive-sequence ISC $i_{+}(t)$ is actually a measure of similarity between the original threephase current and the ideal perfectly balanced one. Similar statements can also be made for the negative- and zero-sequence ISCs. Moreover, in the case of a signal constituted by the sum of several components and considering the linearity property of the ISC transform, it can be concluded that each of the additive components of the original signals is projected on the new axes. In this case, $i_{+}(t)$ contains the sum of the balanced parts of each additive component composing the phase currents, while $i_{-}(t)$ and $i_{0}(t)$ contain the sum of their unbalanced parts. 


\subsection{SNR improvement}

In order to show the signal-to-noise ratio (SNR) improvement provided by the use of the ISC transform, we firstly determine the SNR of the phase currents modeled by Equation (1) considering the given assumptions on the noises. Under these assumptions, the PSD $S_{i_{k}}(f)$ of each phase current can be expressed as in Equation (6), with $\delta\left(f-f_{0}\right)$ denoting a Dirac delta function located at frequency $f_{0}$ and $S_{n_{k}}(f)$ denoting the noise PSDs.

$$
S_{i_{k}}(f)=\left|A_{k}\right|^{2} \delta\left(f-f_{0}\right)+S_{n_{k}}(f)
$$

This equation enables the definition of a SNR for each phase current valid at the frequency of interest $f_{0}$ :

$$
\operatorname{SNR}_{i_{k}}\left(f_{0}\right)=\frac{\left|A_{k}\right|^{2}}{S_{n_{k}}\left(f_{0}\right)}
$$

Using equations (5) and (1), each ISC can be written as:

$$
i_{m}(t)=\left(\sum_{k=1}^{3} \alpha_{m k} A_{k}\right) e^{\mathrm{j} 2 \pi f_{0} t}+\sum_{k=1}^{3} \alpha_{m k} n_{k}(t)
$$

and its power spectral density as:

$$
\begin{aligned}
S_{i_{m}}(f) & =\left|\sum_{k=1}^{3} \alpha_{m k} A_{k}\right|^{2} \delta\left(f-f_{0}\right)+\sum_{k=1}^{3}\left|\alpha_{m k}\right|^{2} S_{n_{k}}(f) \\
& =\left|\sum_{k=1}^{3} \alpha_{m k} A_{k}\right|^{2} \delta\left(f-f_{0}\right)+\frac{1}{9} \sum_{k=1}^{3} S_{n_{k}}(f)
\end{aligned}
$$

where $\left|\alpha_{m k}\right|=\frac{1}{3}$ has been used in the last expression. In a similar way as for phase currents, a SNR at frequency $f_{0}$ can be deduced for each ISC:

$$
\operatorname{SNR}_{i_{m}}\left(f_{0}\right)=\frac{\left|\sum_{k=1}^{3} \alpha_{m k} A_{k}\right|^{2}}{\frac{1}{9} \sum_{k=1}^{3} S_{n_{k}}\left(f_{0}\right)}=\frac{\left|\left\langle\mathbf{a}, \boldsymbol{\alpha}_{m}^{*}\right\rangle\right|^{2}}{\frac{1}{9} \sum_{k=1}^{3} S_{n_{k}}\left(f_{0}\right)}
$$

Equation (7) compares the amount of noise with the fundamental component at frequency $f_{0}$ for one phase current, while Eq. (10) does the same comparison for one ISC, i.e. after the application of the ISC transform. The effect of this transform in terms of signal-to-noise ratio can therefore be summarized thanks to the following SNR gain:

$$
\frac{\operatorname{SNR}_{i_{m}}\left(f_{0}\right)}{\operatorname{SNR}_{i_{k}}\left(f_{0}\right)}=\frac{\left|\left\langle\mathbf{a}, \boldsymbol{\alpha}_{m}^{*}\right\rangle\right|^{2}}{\left|A_{k}\right|^{2}} \times \frac{S_{n_{k}}\left(f_{0}\right)}{\frac{1}{9} \sum_{k=1}^{3} S_{n_{k}}\left(f_{0}\right)}
$$

The first term of the right hand side of Eq. (11) highlights the effect of the transform on the fundamental component of frequency $f_{0}$. More precisely, this term compares its magnitude squared in the ISC $m$ (numerator) and in the phase current $k$ (denominator). The second 
term focuses on the effect of the transform on uncorrelated noises by comparing the noise power spectral density in the ISCs (denominator) and in the phase current $k$ (numerator) at frequency $f_{0}$.

In order to get further insight into the effects of this transform, additional assumptions can be done. Assume for example that the three phase currents contain the same amount of noise at frequency $f_{0}\left(S_{n_{k}}\left(f_{0}\right)=S_{n}\left(f_{0}\right)\right)$, and that their fundamental components are perfectly balanced (as in Equation (2)). In that case, the quantity defined by Eq. (11) can be explicitly expressed for each ISC as:

$$
\begin{gathered}
\frac{\operatorname{SNR}_{i_{+}}\left(f_{0}\right)}{\mathrm{SNR}_{i_{k}}\left(f_{0}\right)}=\frac{\left|\left\langle\mathbf{a}, \boldsymbol{\alpha}_{+}^{*}\right\rangle\right|^{2}}{|A|^{2}} \times \frac{S_{n}\left(f_{0}\right)}{\frac{1}{9} \sum_{k=1}^{3} S_{n}\left(f_{0}\right)}=\frac{|A|^{2}}{|A|^{2}} \times \frac{S_{n}\left(f_{0}\right)}{\frac{1}{3} S_{n}\left(f_{0}\right)}=3 \\
\frac{\mathrm{SNR}_{i_{-}}\left(f_{0}\right)}{\operatorname{SNR}_{i_{k}}\left(f_{0}\right)}=\frac{\operatorname{SNR}_{i_{0}}\left(f_{0}\right)}{\operatorname{SNR}_{i_{k}}\left(f_{0}\right)}=0
\end{gathered}
$$

This means that in this case and at frequency $f_{0}$, the SNR is increased by a factor 3 in $i_{+}(t)$ compared to original phase currents, and that the other two ISCs only contain noise. In other words and as expected, the entire fundamental component of the phase currents is projected onto $i_{+}(t)$ only, while the uncorrelated noises are uniformly spread over the three ISCs. By the same way, if the fundamental component of the three-phase current contains an unbalanced part, this part is entirely projected onto $i_{-}(t)$ and/or $i_{0}(t)$ depending on the type of unbalance. This result is to be related to the one mentioned in [17], where it is shown that for perfectly balanced three-phase signals the Cramer Rao Bound of the frequency estimator is three times lower for the positive-sequence ISC than for single phase approaches.

\subsection{Spectral redundancy for real-valued signals}

In practice, the proposed method is to be applied to real-valued signals which are related to their complex-valued counterparts by the following equation:

$$
\mathbf{i}^{\mathbb{R}}(t)=\frac{1}{2}\left(\mathbf{i}(t)+\mathbf{i}^{*}(t)\right)
$$

When applied to real-valued signals $\left(i_{1}^{\mathbb{R}}(t), i_{2}^{\mathbb{R}}(t), i_{3}^{\mathbb{R}}(t) \in \mathbb{R}\right)$, Eq. (3) leads to ISCs verifying $i_{0}(t) \in \mathbb{R}$ and $i_{+}(t)=i_{-}^{*}(t) \in \mathbb{C}$. In the frequency domain, these two properties express as $I_{0}(f)=I_{0}^{*}(-f)$ and $I_{-}(f)=I_{+}^{*}(-f)$ for $f \in \mathbb{R}$. These two relations highlight a redundancy in the spectral content of ISCs between positive and negative frequencies, which is illustrated in Fig. 1 for the frequency $f_{0}$. From this, it is clear that an exhaustive spectral analysis of a given set of ISCs is obtained by analyzing their spectra only for positive frequencies. This strategy is applied in the rest of this paper where an exhaustive spectral analysis of the ISCs around the stator fundamental frequency $f_{0}$ is obtained by analyzing their spectral content only around $+f_{0}$.

This spectral redundancy of the ISCs leads to one important remark regarding the positive and negative sequence components and analytic signals. That is, while $i_{+}(t)$ and $i_{-}(t)$ 

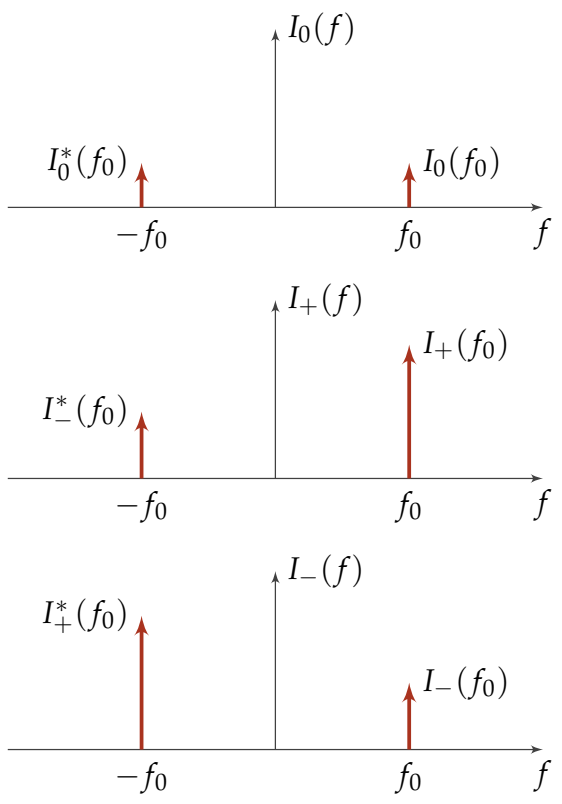

Figure 1: Graphical example of frequency redundancy in ISCs

are complex valued signals, they may have components with negative frequencies and consequently are not analytic signals as graphically shown in Fig. 1. This remark leads to the fact that classical demodulation techniques (i.e. taking the modulus and the derivative of the phase angle) are not defined to be applied directly on the symmetrical components. In other words, instantaneous amplitude and frequency defined on the whole quantity $i_{+}(t)$ do not have a correct physical meaning [18].

\subsection{Simulation results}

In order to illustrate the above presented effects of using the ISC transform, a set of three synthetic phase current signals has been generated based on the formulation of (15). This three-phase signal model, fully justified by the model given in Equation (19) in Section 3 and representative for experimental data presented in Section 4, is based on existing bibliography on mechanical faults signature in single-phase current signals which shows that mechanical faults inducing load torque oscillations lead to phase modulations in current signals [19].

$$
\mathbf{i}^{\mathbb{R}}(t)=\mathbf{i}_{a} \cos \left(2 \pi f_{0} t+\boldsymbol{\varphi}_{A}\right)+\mathbf{i}_{b} \cos \left(2 \pi f_{0} t+\boldsymbol{\varphi}_{B}+p(t)\right)+\mathbf{n}(t)
$$

where:

- $f_{0}=50 \mathrm{~Hz}$ is the fundamental frequency;

- $n_{k}(t), k \in\{1,2,3\}$ are three centered and uncorrelated white Gaussian noises with different variances;

- $\mathbf{i}_{a}$ contains the amplitudes of the non-modulated part of the signals whereas $\boldsymbol{\varphi}_{A}$ contains the phase shifts between their non-modulated parts. These parameters are set 
as $\mathbf{i}_{a}=2 *\left[\begin{array}{lll}0.98 & 1 & 1\end{array}\right]^{T}$ and $\boldsymbol{\varphi}_{A}=\left[\begin{array}{llll}\frac{\pi}{6} & \frac{\pi}{6}-\frac{2 \pi}{3}+\frac{\pi}{12} & \frac{\pi}{6}-\frac{4 \pi}{3}\end{array}\right]^{T}$, thus also comprising a small quantity of unbalance as well as an initial phase shift of $\frac{\pi}{6}$;

- $\mathbf{i}_{b}$ contains the amplitudes of the phase-modulated part of the signals whereas $\boldsymbol{\varphi}_{B}$ contains the corresponding phase shifts. These parameters are set as $\mathbf{i}_{b}=2 *\left[\begin{array}{lll}1 & 1 & 1\end{array}\right]^{T}$ and $\boldsymbol{\varphi}_{B}=\left[\begin{array}{lll}0 & -\frac{2 \pi}{3} & -\frac{4 \pi}{3}\end{array}\right]^{T}$, thus describing a perfectly balanced system of three-phase signals;

- $p(t)$ represents the phase modulation, composed of a sum of two cosines: one cosine at $3 \mathrm{~Hz}$ with a small amplitude such that it can be hidden by noises, and a second cosine at $4 \mathrm{~Hz}$ with an amplitude much higher than the noise levels;

- $f_{s}=1 \mathrm{kHz}$ is the sampling frequency of the generated signals and their duration is 60 seconds.

In the end the ISC transform has been applied to these signals.

Fig. 2 depicts the power spectral densities (PSDs) of the individual phase signals, as well as those of the resulting instantaneous symmetrical components estimated with Welch averaged periodograms using a Hanning window of $2^{14}$ samples, leading to a spectral resolution of approximately $0.12 \mathrm{~Hz}$ clearly sufficient to separate the different components of interest. Several aspects of the ISC approach are highlighted in this figure.

- While the noise floor has different levels on each phase quantity, the three ISCs present an equal lower noise floor, as predicted by (9).

- The unbalance in the phase currents at their fundamental frequency triggers the apparition of the peak at this frequency also in the instantaneous negative- and zerosequence components. However, as the amount of the unbalance is small, the content in $i_{-}(t)$ and $i_{0}(t)$ is much smaller than the one in $i_{+}(t)$.

- The low amplitude modulations of $3 \mathrm{~Hz}$ are not visible in all phase signals PSDs. Due to the improved SNR of the positive-sequence ISC predicted by (11), these modulations are clearly visible in the PSD of $i_{+}(t)$ in Fig. $2 \mathrm{~b}$.

- The amplitude modulations of 3 and $4 \mathrm{~Hz}$ present in each phase signal are only present in the PSD of $i_{+}(t)$ once the ISC transform is applied. This is due to the projection property of the ISC transform, highlighting that only the balanced components of the synthetic phase signals are modulated, while the unbalanced part is not.

In order to quantify the obtained SNR gain for the synthetic signals, the SNR was estimated for each phase current as well as for the positive-sequence component. The noise levels have been estimated based on the obtained PSDs by averaging over the frequency bands which contain only noise $(40-44 \mathrm{~Hz}$ and $56-60 \mathrm{~Hz})$, while the signal power was obtained by summing the PSD under a given peak and the noise under the peak has been 


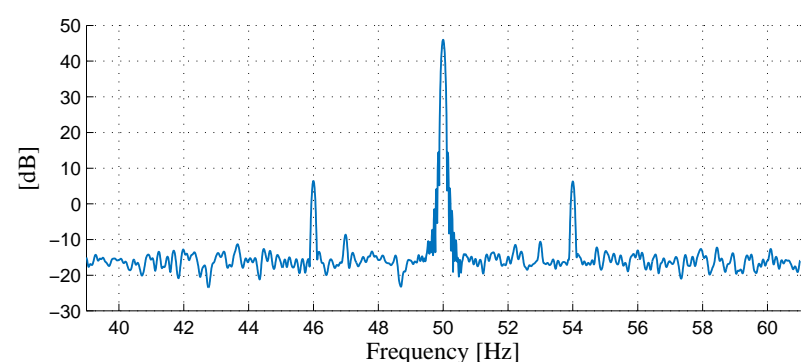

(a) PSD of the current in phase 1

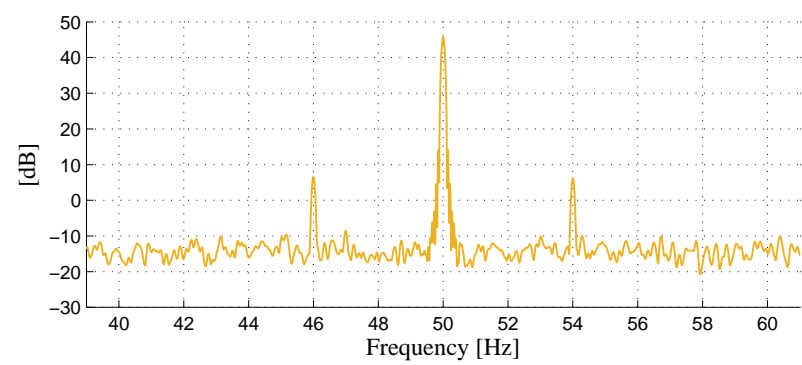

(c) PSD of the current in phase 2

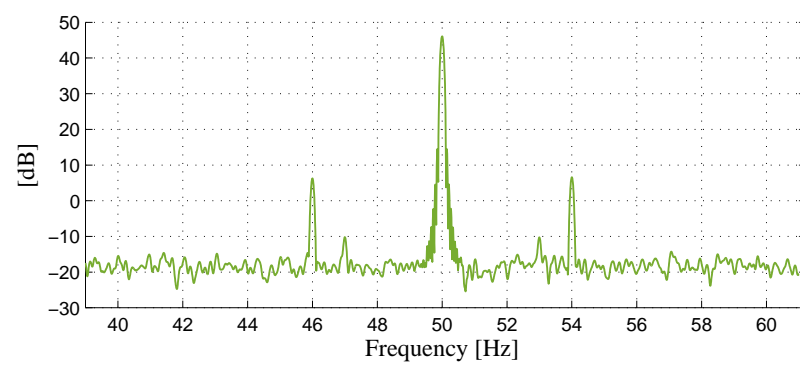

(e) PSD of the current in phase 3

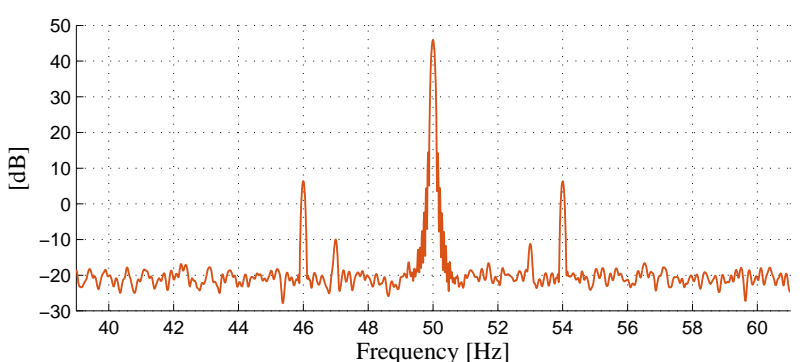

(b) PSD of $i_{+}(t)$

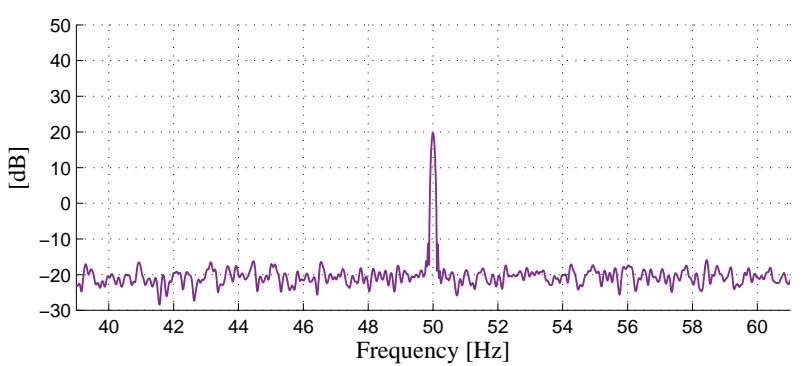

(d) PSD of $i_{-}(t)$

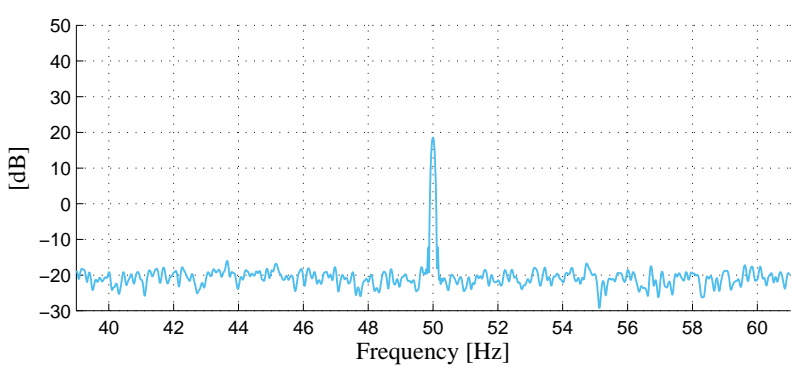

(f) PSD of $i_{0}(t)$

Figure 2: PSDs of the simulated current signals (left: phase quantities, right: instantaneous symmetrical components)

subtracted. The results presented in Table 1 give the local SNR gain around the fundamental frequency.

Table 1 shows that the SNR is indeed improved in the positive-sequence component compared to each of the phase currents. The biggest SNR improvement is with respect to the signal on phase 2, as expected since this corresponds to the poorest SNR of the three phase ones. The lowest gain is obtained with respect to the signal on the third phase, as it can be seen from its PSD in Figure 2 that this signal has the best SNR of the three.

To summarize, the ISC transform defined in Eq. (3) acts differently depending on the nature of the components present in the phase currents at the frequency of interest. The uncorrelated noises are uniformly spread over the three ISCs, while balanced and unbalanced parts are entirely projected onto one specific ISC and by the same way separated from each other. These properties are used in the next section to propose a mechanical fault indicator 
Table 1: Local SNR gains computed for the synthetic signals around $+f_{0}$

$$
\frac{\operatorname{SNR}_{i_{+}}\left(f_{0}\right)}{\operatorname{SNR}_{i_{1}^{\mathbb{R}}}\left(f_{0}\right)}=2.89\left\|\frac{\operatorname{SNR}_{i_{+}}\left(f_{0}\right)}{\operatorname{SNR}_{i_{2}^{\mathbb{R}}}\left(f_{0}\right)}=4.57\right\| \frac{\operatorname{SNR}_{i_{+}}\left(f_{0}\right)}{\operatorname{SNR}_{i_{3}^{\mathbb{R}}}\left(f_{0}\right)}=1.76
$$

taking into account the whole information contained in the three-phase current of a rotating machine.

\section{Mechanical fault indicator}

Models for single-phase current signals have been developed in detail for mechanical faults in induction motors [3]. It has been shown that such faults induce amplitude and/or phase modulations in current signals, depending on the effect of the mechanical fault. In this section the case of phase modulations due to load torque oscillations is detailed. The choice of this model is based on two main reasons: the model is complex enough to demonstrate the various effects of the ISC transform, and it corresponds to the type of fault contained in the experimental data used in Section 4. However, the same algorithm is applicable to the detection of all mechanical faults, regardless of the type. Moreover, the proposed method is based on a signal model containing modulations (due to mechanical faults) rather than on a specific machine model, thus allowing it to be generic in terms of machine type.

\subsection{Signal model}

In case of load torque variations in induction motors, a signal model for the stator current in an arbitrary phase has been proposed in [19] and [3], and is often used to propose and justify mechanical fault indicators. The literature model suggests that in case of mechanical faults which induce load torque variations, the stator current is a sum of two components: a non-modulated component and a modulated one, as expressed in Equation (16).

$$
\begin{aligned}
i^{\mathbb{R}}(t)= & I_{s} \cos \left(2 \pi f_{0} t+\varphi_{s}\right) \\
& +I_{r} \cos \left(2 \pi f_{0} t+\varphi_{r}+\beta \cos \left(2 \pi f_{m} t+\varphi_{m}\right)\right)
\end{aligned}
$$

Eq. (16) shows the two components, where $i^{\mathbb{R}}(t)$ is the stator current in one arbitrary phase, $I_{s}$ and $I_{r}$ are the amplitudes of the current components resulting from the stator and the rotor magnetic fields, $f_{0}$ is the stator fundamental frequency, $\varphi_{s}$ and $\varphi_{r}$ are initial phase shifts and the fault signature is characterized by the phase modulation index $\beta$, its characteristic frequency $f_{m}$ and its initial phase shift $\varphi_{m}$. Obviously, the healthy case is obtained by setting $\beta=0$ in this equation.

The current signal formulation given in Eq. (16) can be rewritten in a more compact form using phasor notations, leading to Equation (17).

$$
i(t)=A e^{\mathrm{j} 2 \pi f_{0} t}+B e^{\mathrm{j}\left(2 \pi f_{0} t+p(t)\right)}
$$

In Eq. (17), the described quantities are:

- $i(t)$ - the current signal in one arbitrary phase; 
- $f_{0}$ - the fundamental frequency;

- $A$ and $B$ - the complex amplitudes of the non-modulated and modulated parts of the signal, containing the amplitude of the respective components, as well as their initial phase;

- $p(t)$ - which models the fault signature and may contain several modulating frequencies (as a sum of cosines for example).

In this equation, the amplitudes and fundamental frequency are constant because the model is developed under the assumption of stationary operating conditions, which has been made for the sake of simplicity.

Furthermore, in order to consider the three signals that can be measured in a three-phase electrical system, (17) can be updated to account for phase specific parameters, as described in (18).

$$
i_{k}(t)=A_{k} e^{\mathrm{j} 2 \pi f_{0} t}+B_{k} e^{\mathrm{j}\left(2 \pi f_{0} t+p(t)\right)}
$$

As $k \in\{1,2,3\}$ denotes the index of each specific phase number, $i_{k}(t)$ represents the signal in each phase. Ideal three-phase systems are defined as being perfectly balanced; however, real systems also contain an inherent unbalance described as small amplitude differences and phase shifts slightly different from the desired $\frac{2 \pi}{3}$ value. Thus, the complex coefficients $A_{k}$ and $B_{k}$ in (18) contain the balanced amounts, as well as the eventual unbalance terms.

For a more compact expression, Eq. (19) gives a vectorial formulation of three-phase currents, where the component $\mathbf{n}(t)$ has been added to model the additive and stationary noise contained in three-phase signals (such as measurement noise). Thus, the signal model proposed by (19) is composed of a periodic part described by the first two factors and a random part described by the noise component.

$$
\mathbf{i}(t)=\mathbf{a} e^{\mathrm{j} 2 \pi f_{0} t}+\mathbf{b} e^{\mathbf{j}\left(2 \pi f_{0} t+p(t)\right)}+\mathbf{n}(t)
$$

In (19), the vector $\mathbf{i}(t)=\left[\begin{array}{lll}i_{1}(t) & i_{2}(t) & i_{3}(t)\end{array}\right]^{T}$ comprises the three current signals, the vectors $\mathbf{a}=\left[\begin{array}{lll}A_{1} & A_{2} & A_{3}\end{array}\right]^{T}$ and $\mathbf{b}=\left[\begin{array}{lll}B_{1} & B_{2} & B_{3}\end{array}\right]^{T}$ contain the complex-valued amplitudes of the non-modulated and phase modulated parts of the signals around the fundamental frequency $f_{0}$ and $\mathbf{n}(t)=\left[\begin{array}{lll}n_{1}(t) & n_{2}(t) & n_{3}(t)\end{array}\right]^{T}$ contains the additive noise present in the three-phase currents.

The two components of frequency $f_{0}$ appearing in Eq. (19) can equivalently be merged into one single component of frequency $f_{0}$ with a time-varying complex amplitude:

$$
\mathbf{i}(t)=\mathbf{c}(t) e^{\mathrm{j} 2 \pi f_{0} t}+\mathbf{n}(t)
$$

where $\mathbf{c}(t)=\mathbf{a}+\mathbf{b} e^{\mathrm{j} p(t)}$. The consequence of Eq. (20) is that electrical signatures of load torque variations in the three phase currents of the machine are both amplitude and phase modulations of the fundamental component when considered as a single sine wave, as already noticed in [19] and [9]. This remark justifies classical demodulation techniques often used 
on phase currents to detect such faults [20]. The goal of this section is now to apply the same strategy to the instantaneous symmetrical components in order to take advantage of their properties. Based on the fact that working three-phase electrical systems are mostly balanced, the positive-sequence ISC of the currents contains most of the information, thus making it a reasonable choice for mechanical fault detection. According to Eq. (20) and (8), $i_{+}(t)$ can be written as:

$$
i_{+}(t)=C_{+}(t) e^{\mathrm{j} 2 \pi f_{0} t}+n_{+}(t)
$$

where $C_{+}(t)$ is a time-varying complex amplitude, with time-varying modulus and phase. This expression shows that as for the phase currents, phase modulations induced by load torque variations are not only visible in the instantaneous phase or frequency of the fundamental component of $i_{+}(t)$, but in its instantaneous amplitude as well. The term $n_{+}(t)$ describing the noise represents the resulting noise after applying the ISC transform on the noise components present in the phase currents.

\subsection{Estimation algorithm}

In order to estimate the instantaneous amplitude and frequency of $i_{+}(t)$ around $f_{0}$ and extract fault signatures, a demodulation step can be used. As detailed in [21], if $i_{+}(t)$ is narrowband i.e. with a spectral content concentrated around $f_{0}$ and a bandwidth much smaller than $f_{0}$, it verifies the Bedrosian theorem and its instantaneous amplitude and phase can be closely estimated through its analytic signal and the Hilbert transform [18]. In the rest of this paper, fault frequencies due to load torque oscillations are assumed to be small compared to $f_{0}$, ensuring that $i_{+}(t)$ is narrowband around $f_{0}$ and that a Hilbert demodulation technique reaches good performance in this case. This remark justifies the structure of the algorithm described in Fig. 3 used to compute the desired fault indicators.

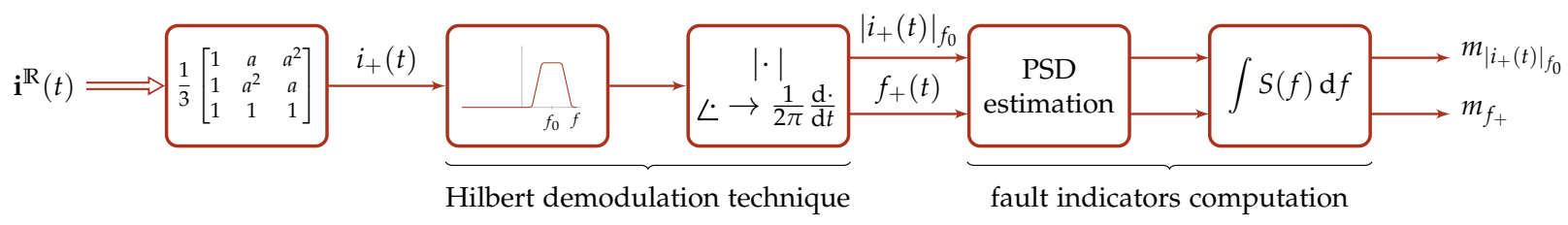

Figure 3: Structure of the algorithm for mechanical fault indicators computation

The first step of this algorithm is the application of the ISC transform defined in Eq. (3) to the phase currents, which is a simple matrix product between the inverse Fortescue matrix and the three-phase measured currents. This first operation returns the three instantaneous symmetrical components corresponding to the three-phase current. The second and third steps consist in applying a Hilbert demodulation technique to $i_{+}(t)$ around $+f_{0}$ in order to estimate the instantaneous amplitude and frequency of its fundamental component. This is realized thanks to a complex-valued frequency-selective filter, with a central frequency $+f_{0}$ and a small bandwidth. The effect of this filter is to leave unchanged the component of $i_{+}(t)$ around $+f_{0}$ and greatly attenuate its other components. As explained in [22], this operation is equivalent to extract the analytic signal of $i_{+}(t)$ around $+f_{0}$ only, and can be 
realized thanks to a simple finite impulse response (FIR) digital filter with complex-valued coefficients, designed using the window method. Based on Eq. (21), the output of this filter mostly consists in $C_{+}(t) e^{\mathrm{j} 2 \pi f_{0} t}$ added to a small residual noise. The instantaneous amplitude and frequency of this analytic narrowband signal is then obtained thanks to its absolute value and the time derivative of its instantaneous phase. The phase time derivative was estimated using a central-difference differentiator, which has the advantage of limiting the amplification of the high-frequency noise due to the differentiation operation. Equation (22) gives the formula for such a differentiator, for a generic discrete signal denoted $x[n]$.

$$
x_{d}[n-1]=\frac{x[n]-x[n-2]}{2}
$$

The last part of the algorithm consists in analyzing the variations of these two quantities and highlighting the fault frequencies to deduce efficient fault indicators. This is realized by first estimating their PSD, and then computing the sum of these PSDs over a given frequency band around fault frequencies. Indeed, the mechanical fault frequencies to be detected are considered to be known, based on system kinematics and operating conditions (i.e. shaft rotating speed). Consequently, by summing the obtained PSDs over a small frequency band $B$ around each fault frequency, eventual faulty components can be detected. A normalized version $m$ of such an indicator [23] is given in (23).

$$
m=\frac{\int_{(B)} S(f) \mathrm{d} f}{\int_{(B)} S_{h}(f) \mathrm{d} f},
$$

where $B$ denotes the chosen frequency band, $S_{h}(f)$ denotes a reference PSD obtained for a healthy system and $S(f)$ is the current PSD obtained for the system in an unknown state. In the case of an healthy condition, such an indicator stays obviously close to one, and tends to increase if faulty components appear in signals.

\subsection{Simulation results}

Two sets of simulations are presented in this section. The first simulation uses the developed signal model for mechanical faults inducing load torque oscillations to show how such fault signatures are visible in the positive-sequence ISC and emphasize the role of the three-phase transform. The second simulation shows the detection capabilities of the proposed fault indicator and compares the results obtained by applying the algorithm on the positive-sequence component to applying it directly on the phase quantities. Both sets of simulations use the signal model for three-phase currents in induction motors given by Equation (15) containing load torque oscillations.

\subsubsection{Mechanical faults inducing load torque oscillations}

In order to illustrate the performance of the proposed approach, the signals previously described in sub-section 2.6 have been further processed. The positive-sequence component has been demodulated using the technique previously described (Fig. 3). In order to compare the results to the ones obtained for single-phase approach, the same processing has been 
applied to each phase signal. The complex-valued filter has been designed using the following characteristics: central frequency of $50 \mathrm{~Hz}$, pass-band of $22 \mathrm{~Hz}$ and transition bandwidth of $4 \mathrm{~Hz}$. After demodulation, the PSDs of the instantaneous amplitude and frequency of the currents positive-sequence ISC, as well as the ones for each phase current, are depicted in Fig. 4. The PSD estimations are obtained with Welch averaged periodograms using the same settings as in sub-section 2.6. As a reminder, the frequencies of interest, which can be assumed to correspond to faults, are 3 and $4 \mathrm{~Hz}$.

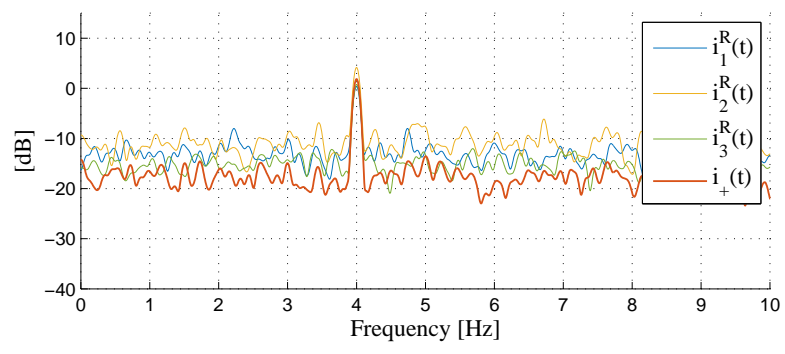

(a) PSD of the instantaneous amplitude

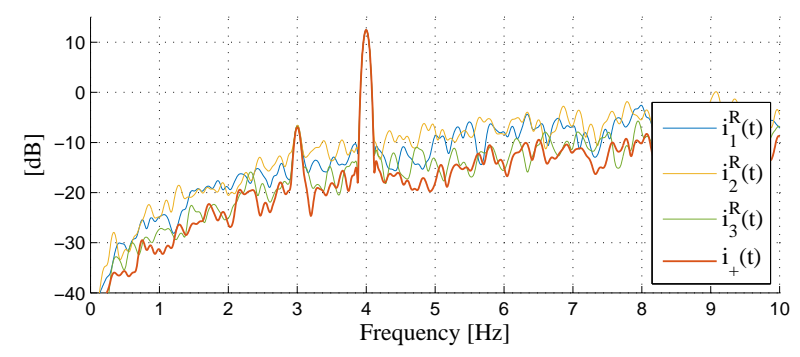

(b) PSD of the instantaneous frequency

Figure 4: PSD of the demodulated $i_{+}(t)$ and each of the three-phase currents

In Fig. 4 it can be observed that for both PSDs the ISCs approach provides better results in terms of SNR, compared to the single-phase approach. As expected considering the different noise levels contained by each simulated current signal, the results depicted in Fig. 4a provide different SNRs for each phase current. Considering the frequency component at $4 \mathrm{~Hz}$, the SNR varies from 12 to $15 \mathrm{~dB}$ for the phase quantities and is $19 \mathrm{~dB}$ for the instantaneous amplitude of the positive-sequence component. The same observation can be made in the PSDs of the instantaneous frequency of the phase currents and the positivesequence component depicted in Fig. 4b. In terms of SNR, the results are different for each phase signal and nonetheless using the instantaneous frequency of $i_{+}(t)$ provides a better SNR, thus the faulty components can be more easily detected.

Finally, the algorithm developed in this section only relies on simple and usual operations such as matrix product, filters and power spectral density estimation and can be simply implemented. Its global structure is similar to the usual current demodulation approaches used for example in [3] or [24], the main difference being that the processed quantity in not one phase current, but the positive-sequence ISC $i_{+}(t)$, giving access to the interesting properties of the ISCs presented in the previous section. It can be noticed that for this particular application, the other two ISCs $i_{-}(t)$ and $i_{0}(t)$ are not useful, but they could be used for different purposes such as stator electrical unbalance characterization as in [23] and [25].

\subsubsection{Detection capabilities of the proposed fault indicators}

In this subsection the detection capabilities of the proposed mechanical fault indicator are evaluated statistically. Moreover, the results of using the positive-sequence ISC are once more compared to the ones obtained using the single-phase quantities. For this statistical 
approach 1000 simulations were performed. For each simulation cycle several sets of signals have been generated using the same model as in (15) using the following different parameters:

- $\mathbf{n}(t)$ have the same standard deviation of 0.15 ;

- The parameters for the non-modulated part are set as $\mathbf{i}_{a}=2 *\left[\begin{array}{lll}1 & 1 & 1\end{array}\right]^{T}$ and $\boldsymbol{\varphi}_{A}=$ $\left[\begin{array}{lll}\frac{\pi}{6} & \frac{\pi}{6}-\frac{2 \pi}{3} & \frac{\pi}{6}-\frac{4 \pi}{3}\end{array}\right]^{T}$, thus perfectly balanced with an initial phase shift of $\frac{\pi}{6}$;

- $p(t)$ represents the phase modulation expressed as $\beta \cos \left(2 \pi f_{m} t\right)$. The modulating frequency $f_{m}$ is equal to $4 \mathrm{~Hz}$. The modulation index $\beta$ takes different values according to Table 2 .

To summarise the main changes from previous paragraph, the three-phase signals are perfectly balanced and they have the same noise levels on each phase. Thus, for each simulation cycle 5 three-phase signals have been generated with the only differences being the random noise (though the noise statistical characteristics have not changed) and the modulation index $\beta$ according to Table 2 .

Table 2: Values of the modulation index for each simulation cycle

\begin{tabular}{|c|c|c|c|c|c|}
\hline & H & S0 & S1 & S2 & S3 \\
\hline \hline$\beta$ & 0 & 0 & 0.002 & 0.004 & 0.006 \\
\hline
\end{tabular}

The first dataset (denoted $\mathrm{H}$ in Table 2) was used to estimate the reference PSD used for the fault indicator. The other 4 sets (denoted starting with the letter $\mathrm{S}$ ) were used to compute the mechanical fault indicator. The parameters for the mechanical fault indicator are: only 1 measurement used for the estimation of the reference PSD and the considered bandwidth $B=4 \pm 0.12 \mathrm{~Hz}$, with $0.12 \mathrm{~Hz}$ being the spectral resolution. Since the three-phase currents are perfectly balanced, only the current signal on phase 1 is used for comparison with $i_{+}(t)$.

As previously mentioned, 1000 such simulations have been performed. For each simulation cycle the mechanical fault indicators' values have been saved and will be used for the statistical analysis. In order to build the Receiver Operating Characteristic (ROC) curves [26] for the indicators the detection thresholds have to be set. The considered thresholds belong to the interval [0 4], with an increment of 0.01 . The value of the indicator surpassing the threshold marks a fault detection. For each threshold for each value of the modulation index (denoted starting with $\mathrm{S}$ in Table 2) the true positive rate was computed as the proportion of detection out of the whole datasets for which the modulation was introduced. The false positive rate was computed using the healthy simulations (denoted S0 in Table 2) and represents the proportion of false detections (the number of detections when there was no modulation over the total number of simulations in healthy case). Figure 5 depicts the obtained ROC curves. The ideal situation is to detect all modulations while never triggering a false alarm. This corresponds to the point $(0,1)$ on the ROC curve.

As the value of the modulation index increases, the ROC curves get closer to the ideal $(0,1)$ point. For the indicators computed using the instantaneous amplitude and especially 

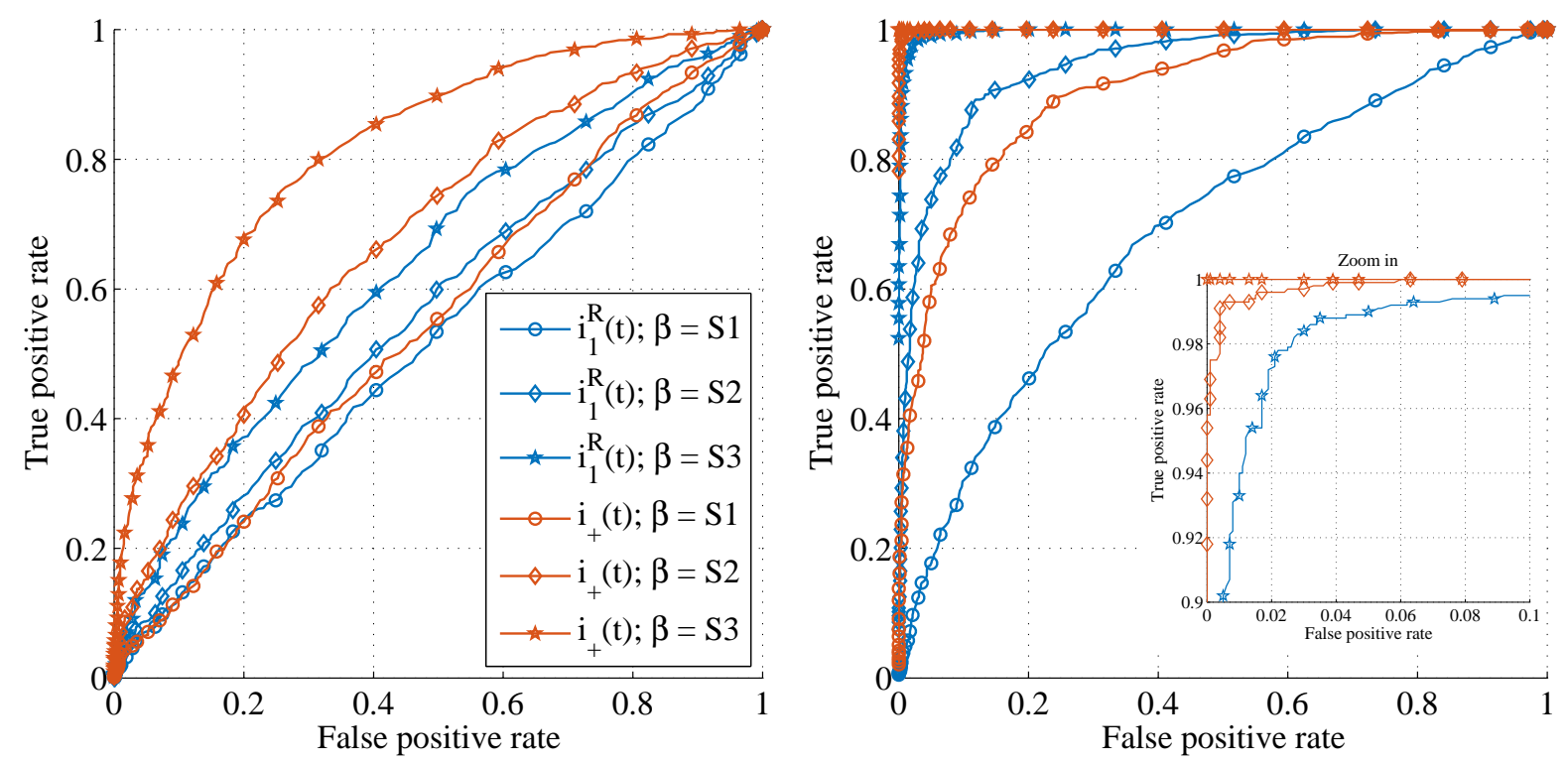

(a) ROC curve for the fault indicators using the IA (b) ROC curve for the fault indicators using the IF

Figure 5: ROC curve for the fault indicators for different values of the modulation index (the false positive rate was estimated using $\mathrm{S} 0$ )

for the lowest values of $\beta$ the detection outcome is approximately random, being located on the main diagonal. However, for each of the values of the modulation index the use of the positive-sequence ISC provides better results than the use of the single current, as expected. By comparing the detection capabilities of the indicators computed using the instantaneous amplitude and instantaneous frequency, it is clear that for this kind of modulations the instantaneous frequency provides better results. Also, the ROC curve for the fault indicators using the instantaneous frequency shows that the detection performance increases with the increase of $\beta$ and that the positive-sequence ISC provides the best results. In order to further validate this approach and demonstrate its good performance, this algorithm is applied on experimental data in the next section.

\section{Experimental results}

\subsection{Experimental set-up}

The proposed condition monitoring method has been tested on experimental data coming from a test-bench purposely designed and developed in the CETIM laboratory (Senlis, France) [27]. The bench emulates the structure and behaviour of a wind turbine, with an electrical motor replacing the wind turbine rotor, followed by a low speed shaft with the main bearing, a gearbox, high speed shaft and three-phase electrical generator (three-phase induction machine SEW Eurodrive DRL160MC4 used as a generator with a maximum power of $10 \mathrm{~kW}$ ), as seen in Fig. 6a. The operating conditions are determined by the speed of the low speed shaft. It should be noted that the test bench generator is not connected to the 
main grid, thus its resulting stator frequency is not imposed by the grid but by the rotating speed of the high speed shaft.

An experiment was conducted for $\approx 200$ hours during which an accelerated deterioration of the main bearing was induced by applying axial and radial forces on the main bearing. Usually the literature presents artificial faults, like for example, drilling a hole of a given diameter into the inner race of a bearing. The advantages of such an approach are that the fault type is known a priori (i.e. inner race fault) and that the values of the fault indicators could be linked to the fault severity. By applying different load forces on the bearing and letting the fault evolve naturally, the outcome of the experiment is not controlled. Otherwise said, one does not necessarily know a priory which type of fault would occur. On the other hand, this approach allows drawing a picture of the fault signature evolution with respect to time.

Throughout the experiment, three-phase current signals have been acquired at the stator of the generator and 21 measurement sets are considered in this paper. The duration of the signals is $\approx 140$ seconds and the sampling frequency of $\approx 1 \mathrm{kHz}$. The signals correspond to stationary operating conditions, with a constant input rotating speed of the low speed shaft of $20 \mathrm{rpm}$.

From the point of view of condition monitoring, the forces applied on the main bearing caused the progressive appearance of an inner race bearing fault (see Fig. 6b) with a corresponding fault frequency of $3.45 \mathrm{~Hz}$, for the considered rotating speed. This fault generates small torque fluctuations on the low speed shaft, which propagate through the gearbox to the high speed and generator shaft and finally lead to small amplitude and frequency modulations in the generator currents. These modulations should be visible in the spectra of the different currents as modulation sidebands located around the fundamental frequency of $67.17 \mathrm{~Hz}$. The advantage of having a fundamental frequency different from $50 \mathrm{~Hz}$ is that the computed indicators are not sensitive to eventual grid disturbances.

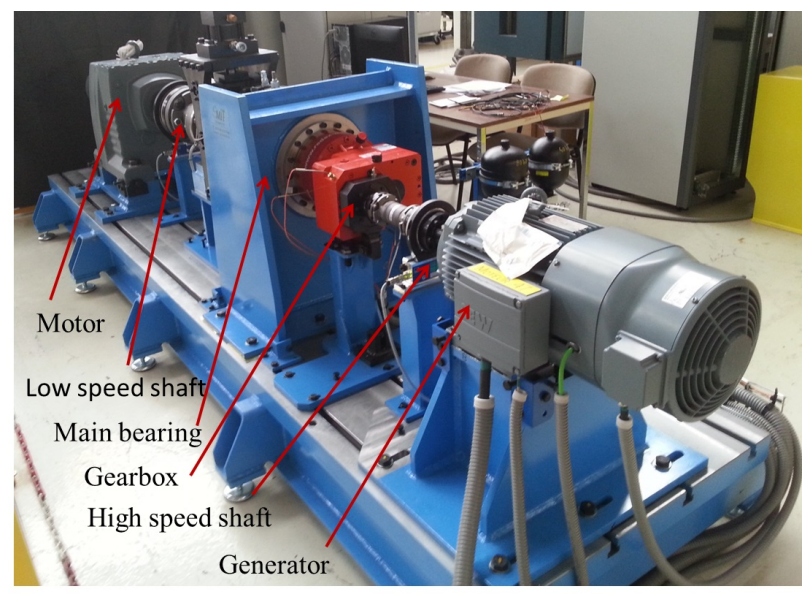

(a) Experimental test-bench

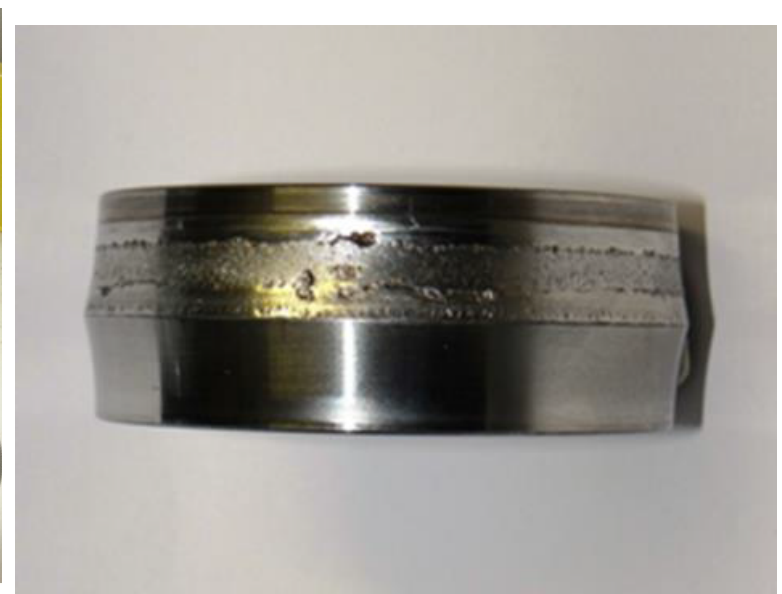

(b) Wide-spread flaking on the main bearing inner race

Figure 6: Experimental setup and resulting bearing fault 


\subsection{Frequency content of the instantaneous symmetrical components}

In this section, the PSDs of the three-phase currents are compared to the frequency content of the computed ISCs. Fig. 7 depicts the PSDs of the experimentally acquired signals, as well as those of the computed ISCs. Each sub-figure contains the superposed PSD obtained for each of the 21 measurements using the same settings as for simulated signals (a Hanning window of $2^{14}$ samples corresponding to a spectral resolution of approximately $0.12 \mathrm{~Hz}$ ). The important points that can be noticed thanks to these experimental results are the following ones.

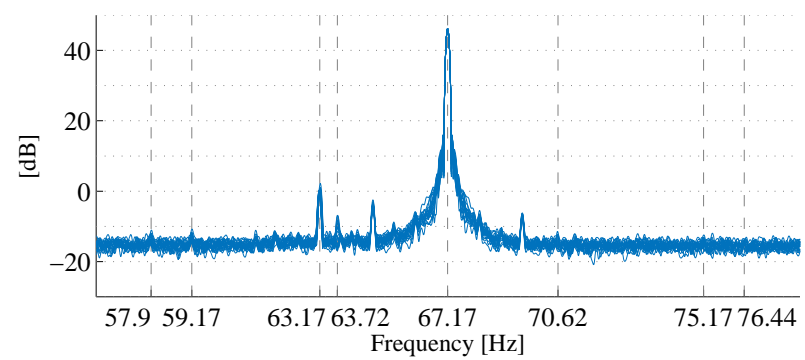

(a) PSD of the current on phase 1

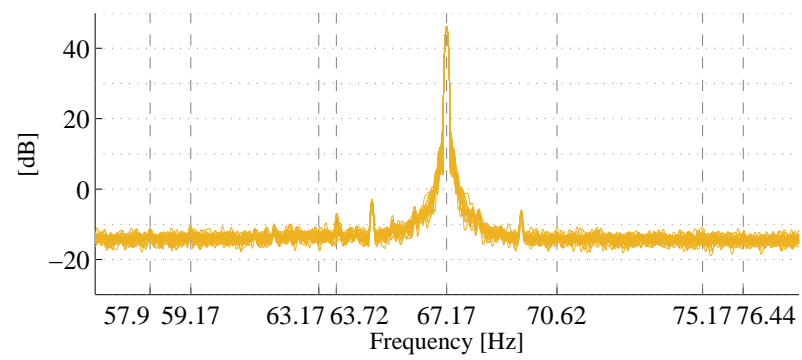

(c) PSD of the current on phase 2

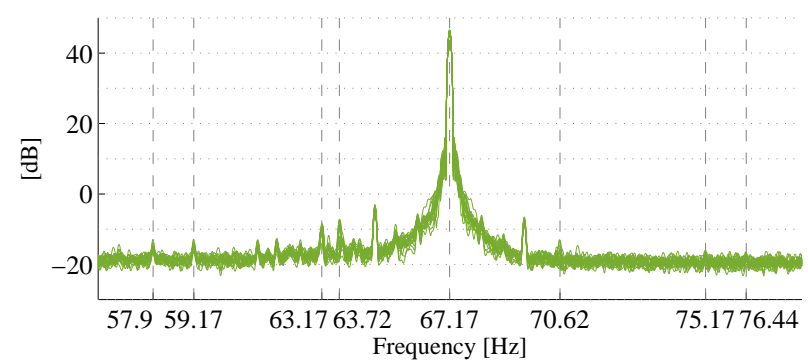

(e) PSD of the current on phase 3

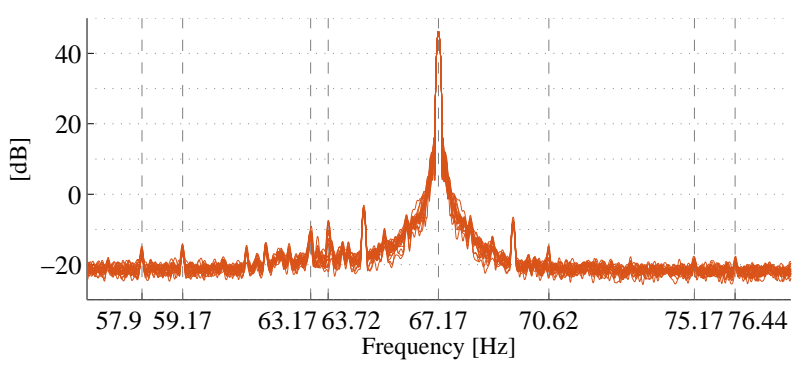

(b) PSD of $i_{+}(t)$

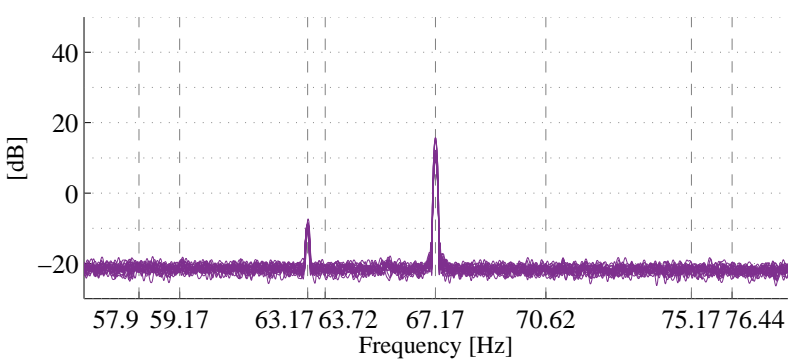

(d) PSD of $i_{-}(t)$

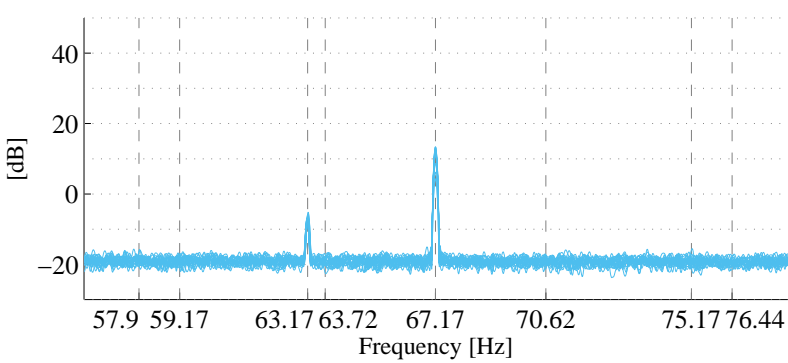

(f) PSD of $i_{0}(t)$

Figure 7: Superposition of the PSDs of the 21 sets of current signals acquired at the output of the generator located on the test-bench (left: phase quantities, right: instantaneous symmetrical components)

- Most of the periodic components are most visible in the PSD of the positive-sequence component. This is particularly true for the two components at $57.9 \mathrm{~Hz}$ and $59.17 \mathrm{~Hz}$ which are almost completely hidden in the noise floor of the PSDs of the current signals 
on phases 1 and 2. This is also the case for the small modulation sidebands located around the fundamental component (at $63.72 \mathrm{~Hz}$ and $70.62 \mathrm{~Hz}$ ), which should contain the bearing fault signature. This highlights their balanced nature, and the ability of the ISC transform to improve the SNR regarding these balanced components.

- Some periodic components are partly unbalanced. For example, the presence of the fundamental component in the PSDs of the negative- and zero-sequence components indicates the presence of an electrical unbalance in the three-phase system. However, their small magnitudes could be accounted for by a natural inherent unbalance present in all real three-phase systems. Another case is the component at $63.17 \mathrm{~Hz}$, which is only present in two of the three phase currents and therefore clearly visible in the negative- and zero-sequence components. This result highlights the ability of the ISC transform to separate balanced and unbalanced parts of the different components constituting the signals.

- The noise levels on $i_{+}(t)$ and $i_{-}(t)$ are equal, as expected for uncorrelated phenomena. However, the slightly increased level on $i_{0}(t)$ suggests that there is a small correlated synchronous noise in the three phase currents. This might be due to a small amount of crosstalk usually present in synchronous multichannel acquisition systems.

\subsection{Mechanical fault indicators}

Based on the ISC approach, the positive-sequence ISC of the currents is demodulated according to the algorithm depicted in Section 3.2. The complex-valued filter has a central frequency of $67.17 \mathrm{~Hz}$ equal to the fundamental frequency, its passband is of $22 \mathrm{~Hz}$ and transition band of $4 \mathrm{~Hz}$. Considering the stationarity of the operating conditions, two mechanical fault indicators are computed by summing the PSD of the obtained instantaneous amplitude and frequency around the fault frequency of $3.45 \mathrm{~Hz}$ over a frequency band equal to $0.12 \mathrm{~Hz}$, the spectral resolution. In case of small non-stationarity of the operating conditions like in [28], the proposed indicators can be easily adapted by increasing the frequency band over which they are computed. The healthy condition estimate is obtained by averaging the signal power under the peak for the first 5 measurements, for each of the considered quantities individually. In order to achieve the comparison with a usual single-phase approach, the same processing is applied directly on the phase currents, leading to three additional fault indicators.

Fig. 8 depicts the obtained results. Firstly, one can observe that the use of the instantaneous frequency provides better overall results for this type of fault, compared to the instantaneous amplitude. Secondly, the use of the positive-sequence component gives better results than considering each phase signal individually. This result is expected due to the improved SNR obtained when the whole three-phase currents are considered, rather than single phase. Due to its poorest SNR, the current signal on phase 2 provides the worst results and does not enable the detection of the mechanical fault in the case of a single-phase approach.

Another interesting remark concerning Figure 8 is that it shows the time evolution of the fault signature in electrical signals throughout the experiment. Because no maintenance 

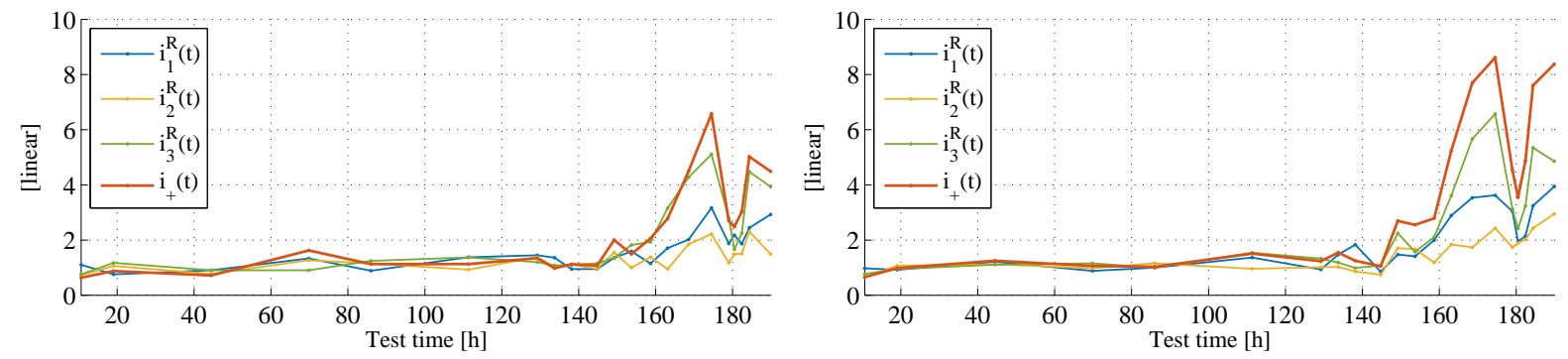

(a) Fault indicator using the instantaneous amplitude(b) Fault indicator using the instantaneous frequency

Figure 8: Mechanical fault indicators computed from the currents signals (over the frequency band $B=$ $3.45 \pm 0.06 \mathrm{~Hz})$

work has been done during the experiment, a continuous increase of the deterioration of the bearing is expected. However, the fault signature does not show a continuous increase, especially around the $180^{\text {th }}$ hour of the experiment. This remark highlights the fact that the fault indicator describes the signature profile of the load torque oscillations induced by the mechanical faults and not the fault severity itself.

\section{Conclusions}

In this paper a method for mechanical fault detection in electrical rotating machines using three-phase electrical signals has been presented. The method is based on a signal model which takes into account the multi-component nature of current signals, as well as the use of instantaneous symmetrical components. More specifically, the instantaneous amplitude and frequency the positive-sequence ISC are used for mechanical faults detection. For the demodulation step the proposed method uses a complex-valued filter to select only the positive frequencies present in the positive-sequence ISC. The computation of the mechanical fault indicators is then based on the demodulated quantities. However, one limitation of this approach is given by the bandwidth of the employed filter which limits the maximum value of the detectable fault frequency.

The three main advantages of using symmetrical components compared to single-phase quantities are: the use of the whole information contained in three-phase electrical systems, the separation of balanced and unbalanced parts of three-phase signals, and the improvement of the signal-to-noise ratio. This paper focuses on the latter, theoretically showing that the SNR is improved, as well as validating the results using both synthetic and experimental signals. As a direct consequence of the SNR improvement, the proposed fault indicators based on ISCs offer better detection capabilities than the ones based on single-phase approaches. Another positive point is that the proposed algorithm is well suited to on-line implementation on an embedded condition monitoring system due to its employment of low complexity operations.

The method proposed for detection of mechanical faults is particularly suitable for threephase induction machines as shown in this article, and a dedicated study is still to be done 
for other types of machines (such as synchronous machines or others). The proposed fault indicator relies on the phase modulations of the fundamental component of three-phase stator signals. The type of connection of the stator windings $(\Delta$ or $Y$ ) does not limit the validity of the approach since it only influences the maximum amplitude and original phase shift of these components and not their modulations. Moreover, the method focuses on the fundamental component only and filters out every other component. Consequently, using a machine connected to the network directly or to an inverter would not influence the results, considering that only the fundamental frequency is used.

In terms of mechanical fault indicator, there are several perspectives for improvement. Indeed, while the proposed algorithm can still be employed in case of small non-stationarity by increasing the frequency band over which the indicator is computed, in case of large variations in operating conditions the last step relying on spectral analysis must be adapted to non-stationary signals, for example by using time-frequency representations. However, the general principle of using the ISCs and their advantages instead of phase or line quantities still stands. Also, the case of faults generating modulations of electrical signals with a frequency higher than the fundamental frequency will be included in future works.

\section{Acknowledgments}

This research has been partly supported by KIC InnoEnergy, a company supported by the European Institute of Innovation and Technology (EIT), through KAStrion European project.

The authors would like to thank CETIM (Centre Technique des Industries Mécaniques) for providing the test-bench and experimental data.

\section{References}

[1] H. A. Toliyat, S. Nandi, S. Choi, H. Meshgin-Kelk (Eds.), Electric machines: modeling, condition monitoring, and fault diagnosis, CRC Press, 2013.

[2] M. Blodt, J. Regnier, J. Faucher, Distinguishing load torque oscillations and eccentricity faults in induction motors using stator current wigner distributions, IEEE Transactions on Industry Applications 45 (6) (2009) 1991-2000. doi:10.1109/TIA.2009.2031888.

[3] M. Blodt, P. Granjon, B. Raison, J. Regnier, Mechanical fault detection in induction motor drives through stator current monitoring - theory and application examples, in: W. Zhang (Ed.), Fault Detection, InTech, 2010, pp. 451-487.

[4] C. L. Fortescue, Method of symmetrical co-ordinates applied to the solution of polyphase networks, Transactions of the American Institute of Electrical Engineers XXXVII (2) (1918) 1027-1140. doi: 10.1109/T-AIEE. 1918.4765570.

[5] E. Clarke, Circuit Analysis of A-C Power Systems, New York : J. Wiley \& Sons, Inc., 1943.

[6] G. C. Paap, Symmetrical components in the time domain and their application to power network calculations, IEEE Transactions on Power Systems 15 (2) (2000) 522-528. doi:10.1109/59.867135.

[7] J. L. H. Silva, A. J. M. Cardoso, Bearing failures diagnosis in three-phase induction motors by extended Park's vector approach, in: 31st Annual Conference of IEEE Industrial Electronics Society, 2005. IECON 2005., 2005, pp. 6 pp.-- doi:10.1109/IECON.2005.1569315.

[8] E. Fournier, A. Picot, J. Regnier, M. T. Yamdeu, J.-M. Andrejak, P. Maussion, Current-based detection of mechanical unbalance in an induction machine using spectral kurtosis with reference, IEEE Transactions on Industrial Electronics 62 (3) (2015-03) 1879-1887. doi:10.1109/TIE. 2014.2341561. 
[9] B. Trajin, M. Chabert, J. Regnier, J. Faucher, Hilbert versus Concordia transform for three-phase machine stator current time-frequency monitoring, Mechanical Systems and Signal Processing 23 (8) (2009) 2648-2657. doi:10.1016/j.ymssp.2009.05.015.

[10] Y. Amirat, V. Choqueuse, M. E. H. Benbouzid, Wind turbines condition monitoring and fault diagnosis using generator current amplitude demodulation, in: Energy Conference and Exhibition (EnergyCon), 2010 IEEE International, 2010, pp. 310-315. doi:10.1109/ENERGYCON. 2010.5771697.

[11] V. Choqueuse, M. E. H. Benbouzid, Y. Amirat, S. Turri, Diagnosis of three-phase electrical machines using multidimensional demodulation techniques, IEEE Transactions on Industrial Electronics 59 (4) (2012-04) 2014-2023. doi:10.1109/TIE.2011.2160138.

[12] E. H. El Bouchikhi, V. Choqueuse, M. Benbouzid, J. A. Antonino-Daviu, Stator current demodulation for induction machine rotor faults diagnosis, in: Green Energy, 2014 International Conference on, 2014, pp. 176-181. doi:10.1109/ICGE. 2014.6835418.

[13] Y. Xia, S. Douglas, D. Mandic, Adaptive Frequency Estimation in Smart Grid Applications: Exploiting Noncircularity and Widely Linear Adaptive Estimators, IEEE Signal Processing Magazine 29 (5) (2012) 44-54. doi:10.1109/MSP.2012.2183689.

[14] Y. Xia, D. P. Mandic, Widely Linear Adaptive Frequency Estimation of Unbalanced Three-Phase Power Systems, IEEE Transactions on Instrumentation and Measurement 61 (1) (2012) 74-83. doi: 10.1109/TIM.2011.2159409.

[15] M. B. K. Bouzid, G. Champenois, New expressions of symmetrical components of the induction motor under stator faults, IEEE Transactions on Industrial Electronics 60 (9) (2013) 4093-4102. doi:10. 1109/TIE. 2012.2235392.

[16] W. V. Lyon, Transient Analysis of Alternating-current Machinery: An Application of Method of Symmetrical Components, Technology Press of Massachusetts Institute of Technology, John Wiley and Sons, New York, 1954.

[17] V. Choqueuse, E. Elbouchikhi, M. Benbouzid, Maximum likelihood frequency estimation in smart grid applications, in: 2015 IEEE 24th International Symposium on Industrial Electronics (ISIE), 2015, pp. 1339-1344. doi:10.1109/ISIE.2015.7281667.

[18] B. Boashash, Estimating and interpreting the instantaneous frequency of a signal. I. Fundamentals, Proceedings of the IEEE 80 (4) (1992) 520-538. doi:10.1109/5.135376.

[19] M. Blodt, M. Chabert, J. Regnier, J. Faucher, Mechanical load fault detection in induction motors by stator current time-frequency analysis, IEEE Transactions on Industry Applications 42 (6) (2006) 1454-1463. doi:10.1109/TIA.2006.882631.

[20] S. H. Kia, H. Henao, G. A. Capolino, Efficient digital signal processing techniques for induction machines fault diagnosis, in: Electrical Machines Design Control and Diagnosis (WEMDCD), 2013 IEEE Workshop on, 2013, pp. 232-246. doi:10.1109/WEMDCD.2013.6525183.

[21] B. Picinbono, On instantaneous amplitude and phase of signals, IEEE Transactions on Signal Processing 45 (3) (1997) 552-560. doi:10.1109/78.558469.

[22] A. Reilly, G. Frazer, B. Boashash, Analytic signal generation-tips and traps, IEEE Transactions on Signal Processing 42 (11) (1994) 3241-3245. doi:10.1109/78.330385.

[23] G. Cablea, P. Granjon, C. Bérenguer, P. Bellemain, Online condition monitoring of wind turbines through three-phase electrical signature analysis, in: CD proceedings of the Twelfth International Conference on Condition Monitoring and Machinery Failure Prevention Technologies, 2015.

[24] R. Puche-Panadero, M. Pineda-Sanchez, M. Riera-Guasp, J. Roger-Folch, E. Hurtado-Perez, J. PerezCruz, Improved resolution of the MCSA method via Hilbert transform, enabling the diagnosis of rotor asymmetries at very low slip, IEEE Transactions on Energy Conversion 24 (1) (2009) 52-59. doi: 10.1109/TEC. 2008.2003207.

[25] G. Cablea, P. Granjon, C. Bérenguer, Method for computing efficient electrical indicators for offshore wind turbine monitoring, Insight - Non-Destructive Testing and Condition Monitoring 56 (8) (2014) 443-448. doi:doi:10.1784/insi.2014.56.8.443.

[26] S. M. Kay, Detection theory, 1st Edition, Vol. 2 of Fundamentals of statistical signal processing, Prentice Hall PTR, Upper Saddle River, NJ, 1998. 
[27] N. Bédouin, S. Sieg-Zieba, Endurance testing on a wind turbine test bench. A focus on slow rotating bearing monitoring, in: CD proceedings of the Twelfth International Conference on Condition Monitoring and Machinery Failure Prevention Technologies, 2015.

[28] G. Cablea, P. Granjon, C. Bérenguer, Advances in Condition Monitoring of Machinery in NonStationary Operations: Proceedings of the Fourth International Conference on Condition Monitoring of Machinery in Non-Stationary Operations, CMMNO'2014, Lyon, France December 15-17, Springer International Publishing, Cham, 2016, Ch. Method of Analysing Non-stationary Electrical Signals, pp. 3-14. doi:10.1007/978-3-319-20463-5_1. 